\section{Relationships between clinical measures of visual function and anatomic changes associated with bevacizumab treatment for choroidal neovascularization in age-related macular degeneration}

YB Ünver ${ }^{1}$, GA Yavuz ${ }^{2}$, N Bekiroğlü ${ }^{3}$, P Presti $^{4}$, W Li ${ }^{5}$ and $\mathrm{SH}$ Sinclair ${ }^{5,6}$

\section{(and SH Sinclairs,}

${ }^{1}$ Beyoğlu Eye Training and Research Hospital, İstanbul, Turkey

${ }^{2}$ Ophthalmology

Department, Medical School, Düzce University, Düzce, Turkey

${ }^{3}$ Biostatistics Department, Medical School, Marmara University, İstanbul, Turkey

${ }^{4}$ Interactive Multi-Media Technology Center, Georgia Institute of Technology, Atlanta, GA, USA

coherence tomography.

Results Logistic regression analysis showed an association between the vision outcomes of EDTRS letter and $\mathrm{BA}^{\circ}$ with the change in SRF thickness ( $R^{2}: 0.47$ and 0.35 , respectively). The outcome of the vision measurement of GMA was associated with the change in SRF thickness, in CNV thickness, and in CNV fibrosis grade $\left(R^{2}: 0.34\right)$. No association was noted between the outcomes of ETDRS line with the change in any anatomic outcomes. Conclusion Acuity perimetry outcomes in this study seemed to offer improved understanding of the relationship between the vision outcomes and the measured anatomic changes. It seemed that neither ocular coherence tomography nor fluorescein angiography alone offered sufficient morphologic markers for prediction of functional outcomes.

Eye (2009) 23, 453-460; doi:10.1038/eye.2008.349; published online 28 November 2008

Keywords: age-related macular degeneration; anatomic outcome; bevacizumab; avastin treatment; vision outcome; central field acuity testing
${ }^{5}$ Department of Ophthalmology, Medical School, Drexel University, PA, USA

${ }^{6}$ Vimetrics, LLC, Media, PA, USA

Correspondence: YB Ünver, Beyoğlu Eye Training and Research Hospital, 311 E. Baltimore Avenue 1st floor,

Suite 100, Media,

Kuledibi,

Istanbul, Pa. 19035,

Turkey

Tel: + 610892 1708; Fax: + 902164674280

E-mail: yaprakbanu@ gmail.com

Received: 9 July 2008 Accepted in revised form: 13 October 2008

Published online:

28 November 2008

This study was presented in 2008 ARVO meeting. grading of CNV leakage on fluorescein angiography, and central retinal PED, and 


\section{Background}

Choroidal neovascularization (CNV) accounts for $75 \%$ or more of the eyes that suffer severe vision loss with age-related macular degeneration (AMD). ${ }^{1}$ The vision loss appears due to sero-sanguinous leakage into the subretinal space, with resultant large scotomas caused by toxic intra and subretinal fluid (SRF), fibrotic organization and atrophy of retina, retinal pigment epithelium (RPE), and choriocapillaris. Within the past decade, new pharmaceutical therapies have focused on reducing the leakage of the $\mathrm{CNV}$ with hopefully less resultant fibrosis of the $\mathrm{CNV}$ and less $\mathrm{RPE}$, and choriocapillaris or retinal atrophy. Most recently, anti-VEGF therapy seems to be the most promising with bevacizumab showing significant efficacy to cause regression of the CNV. ${ }^{2,3}$ Indications for treatment, retreatment, and/or surveillance have depended mainly upon the evaluation of $\mathrm{CNV}$ leakage and have used visual acuity as the outcome determinant.

Fundus photography (FP) and fluorescein angiography (FA) - for more than three decades - have provided the major information about $\mathrm{CNV}$ composition, size, and leakage, whereas some studies have suggested that angiographic parameters do not adequately explain variations in visual acuity. ${ }^{4-6}$ Moutray et al, ${ }^{7}$ in a crosssectional study, observed significant correlation between distance VA and near-word acuity with angiographic parameters such as CNV diameter and area, and leakage area.

More recently, optical coherence tomography (OCT) has gained popularity to evaluate the severity of exudative leakage by evaluating the extent of SRF or retinal thickening. ${ }^{8}$ Recent cross-sectional trials of AMD$\mathrm{CNV}$ treatment have indicated that OCT measurements, such as the CNV thickness, foveal thickness, and maximum retinal thickness, are correlated with visual acuity, ${ }^{4,9-11}$ but the associations were weak, whereas others reported minimal or inconsistent correlations. ${ }^{7}$ In other longitudinal studies, OCT parameters, such as maximum retinal thickness, foveal thickness, or cystoid changes, were associated with decreased distance VA at baseline and follow-up. ${ }^{5,12}$

Vision outcomes resulting from the treatment of AMD$\mathrm{CNV}$ have been evaluated primarily by using the ETDRS $0.1 \log$ MAR chart. ${ }^{8-10}$ However, visual acuity is recognized to have significant limitations in evaluating vision, especially in the eye with macular disease. The measurement of acuity using such a chart, it should be remembered, defines only the ability to discriminate high contrast letters only under the lighting level in the examination lane and only within a small area of the visual field, and not necessarily at fixation but often at another preferred fixation locus. High contrast VA does not evaluate the limits of vision under conditions that are associated with activities of daily living. ${ }^{11}$ The evaluation of vision parameters other than distance acuity has been limited. Moutray ${ }^{7}$ evaluated near word acuity and Pelli-Robson contrast sensitivity, and found stronger relationships with the anatomic parameters than distance VA. However, these chart tests suffer many of the same limitations as with distance VA cited above.

A newly developed central field testing device (Field Acuity Analyzer (FAA) Vimetrics, Media, PA, USA) allows the evaluation of the discrimination capabilities of vision in the central $20^{\circ}$ diameter field under varying conditions of target hue, contrast, and background illumination. ${ }^{13}$ The device was developed to improve the measurement of visual performance in eyes with macular disease under luminance, hue, and contrast conditions that simulate conditions encountered in normal tasks of daily living.

This pilot study examines the relationships between measures of vision, including the ETDRS chart acuity and FAA field acuity parameters, with measurements of leakage, hemorrhage, atrophy, and fibrosis obtained from FP and FA, as well as with parameters of SRFand fibrosis measured from OCT performed on eyes undergoing bevacizumab treatment for $\mathrm{CNV}$ due to AMD.

\section{Methods}

A retrospective review of 50 eyes of 48 patients was conducted with AMD-CNV who had undergone intravitreal bevacizumab injections, $1.25 \mathrm{mg}$ in $0.05 \mathrm{ml}$, at 6-week intervals with at least three injections and 6 months follow-up after the initial injection. All patients were informed and consent was obtained for the off-label use of bevacizumab and its potential benefits and complications, as well as for the additional testing. Bestcorrected VA was performed before the first injection and at each 6-week visit, recording the smallest line in which at least three of the five Sloan letters were correctly read (ETDRS line), as well as the total letter score (ETDRS letter). ${ }^{14} \mathrm{FP}$ and FA, OCT, and FAA acuity perimetry also were performed, before and at 6 weeks following each of the three bevacizumab injections. Eyes that showed CNV due to AMD with the greatest linear dimension less than $5400 \mu \mathrm{m}$ and with an ETDRS line acuity worse than $20 / 25$, but better than $20 / 320$, and which was determined to be caused by maculopathy, were included. Eyes that were exhibiting significant lens opacity or optic nerve pathology, which was sufficient to affect vision, were excluded. 


\section{Testing}

Fundus photography and fluorescein angiography FP and FA were performed using a standard clinical protocol with stereoscopic capture of digital images. Image pairs were viewed stereoscopically to measure or grade the following:

(1) Greatest linear dimension of the CNV was measured including all classic and occult portions, and all contiguous elements that could obscure the margins (such as overlying hemorrhage).

(2) The area of any pigment epithelial detachment (PED) contiguous with the CNV was estimated in the disc area.

(3) The leakage of the CNV was graded 0-4.

(4) The severity of RPE atrophy was graded 0-3.

(5) The severity of CNV fibrosis was graded $0-3$.

(6) The severity of subretinal hemorrhage was graded $0-2$.

\section{Ocular coherence tomography}

Ocular coherence tomography scans were obtained using the OCT II (Ocular Technologies Inc., Toronto, Canada). A single, experienced technician obtained the OCT scans according to a standardized protocol. Initially, a horizontal line scan of $7 \mathrm{~mm}$, positioned on the equator of the optic disc and offset by $5^{\circ}$, was acquired to locate the fovea and for reproducibility. A raster of horizontal line scans, $6 \mathrm{~mm}$ long with $1-3 \mathrm{~mm}$ intervals, were used to scan the entire lesion, with a minimum of 3 to a maximum of 10 , depending on the vertical span of the lesion. The optimal scan with a clear image of the foveal contour was selected for measurement according to methods defined by Hee. ${ }^{8,15}$ Maximum CNV thickness was measured along with central foveal thickness, maximum SRF thickness, and maximum PED thickness.

All FP, FA, and OCT measurements were performed by a single, experienced retina specialist without the knowledge of the stage of treatment or the vision measurements.

An anatomic successful outcome of treatment was defined as one or more of the following:

(1) A minimum of $300 \mu$ decrease in CNV diameter

(2) A minimum of $300 \mu$ decrease in PED diameter

(3) A decrease in CNV leakage of at least 2 grade levels

(4) Minimal fibrosis ( $\leqslant$ grade 1 ) at follow-up

(5) No hemorrhage at follow-up

(6) No change in the RPE atrophy grading

(7) A minimum of $100 \mu$ decrease in foveal thickness.

(8) No SRF at follow-up

(9) No PED at follow-up or a $\geqslant 50 \mu$ decrease in PED thickness

(10) $\geqslant 100 \mu$ decrease in CNV thickness.

\section{Field acuity perimetry}

Acuity perimetry was performed using a prototype FAA system, in which Landolt $C$ optotypes were presented on an LCD monitor $(1280 \times 1024$ screen resolution,

Princeton Graphics, CA, USA) placed at a distance of $86 \mathrm{~cm}$ from the patient's eye (Figure 1). At this distance, the monitor subtends a visual angle of $18^{\circ}$ vertically and $22^{\circ}$ horizontally. The patient was seated with his (her) head stabilized in a chin rest and forehead strap, and was given his (her) full optical correction for the monitor distance. The treated eye was tested with the fellow eye occluded. Subjects were instructed to fixate on a central, red, cross target throughout the test. High contrast discrimination was tested at fixation and then at 25 intercepts spaced throughout the visual field up to $10^{\circ}$ eccentricity. The discrimination threshold was determined by the patient's ability to determine the correct orientation of a white, Landolt $C$ presented for $250 \mathrm{~ms}$ duration, with the centroid at each field intercept and randomly tumbled in one of four cardinal orientations. The patient's responses were recorded from a hand-held pad that contained four buttons with which they indicated the direction of the opening of the Landolt $C$ after each presentation. The test was conducted in a darkened room with the optotype representing a 99.3\% Michelson contrast (Monaco-optix, Monaco Systems Inc., Andover, MA, USA) against a black pedestal and background,

both of $1 \mathrm{Cd} / \mathrm{M}^{2}$. The pedestal was $2-4$ times the size of

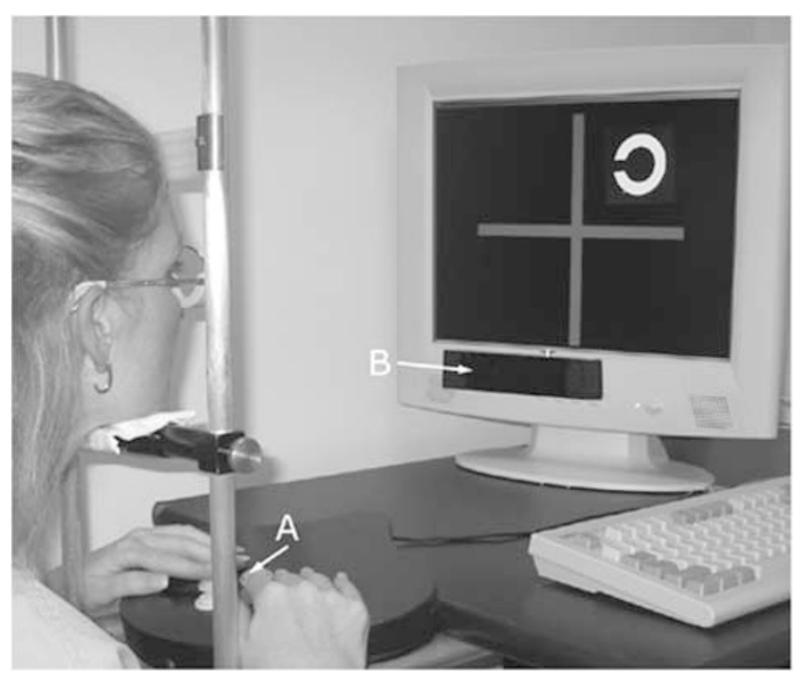

Figure 1 Testing apparatus: patient in chin rest is tested monocularly with appropriate correction and for screen distance. The patient signals their response to orientation of a tumbled Landolt $C$ that is flashed on screen by pressing one of four buttons on the button pad (A). Fixation is monitored with an infrared pupil centrum and corneal reflex camera tracking system (B) mounted in box on bezel of monitor. 
the letter. Eye fixation was monitored throughout the test (to within $1^{\circ}$ ) by the use of an infrared illumination and camera system with measurements at $30 \mathrm{~Hz}$ (I-Scan, Cambridge, Mass, Figure 1). Eye position and deviation were first monitored during the testing of the Landolt $C^{\prime} s$ presented at fixation; thereafter, when the peripheral intercepts were tested, if fixation deviated during each presentation by more than that recorded during the testing at fixation, the response was discarded and later retested.

Before testing on all occasions, a standard set of instructions is presented by the program along with presentation of a trial test to facilitate understanding and participation. The Landolt $\mathrm{C}$ was first presented at fixation with an optotype size of 25 arc min, and then, depending upon the response, the program reduced or enlarged the optotype size in standard ETDRS chart 0.1 $\log$ MAR steps until a threshold was reached, defined as the smallest-sized letter for which a correct response was elicited with two incorrect responses at the next smaller size. The smallest Landolt $C$ size that could be presented, given the pixel density of the screen, subtended 5 arc min $(20 / 20)$, and the largest 80 arc min (20/320). After thresholding for the smallest optotype discriminated at fixation, the Landolt C's were presented and thresholded with the same techniques at each of the peripheral intercepts, with presentations occurring randomly among the intercepts. Intervals between presentations were adjusted to the person's responses, ranging from 1 to $4 \mathrm{~s}$. Average performance time was $8.45 \mathrm{~min}$ (range 8-10) per eye. Every patient who was conducted to the FAA was able to finish the test. Test-retest reliability of the FAA was found to be as good as the test-retest reliability of ETDRS.

Two parameters were recorded from the results of the perimetry: (1) the best acuity thresholded at any intercept within $6^{\circ}$ of fixation (BA6 ${ }^{\circ}$ ) expressed in logMAR and (2) the global macular acuity (GMA), an average of all intercepts within $10^{\circ}$, weighted for proximity to fixation, also expressed in log MAR.

A successful vision outcome following treatment was defined as one or more of the following change in measured visual function:

(1) Three lines (0.3 logMAR) or more improvement in ETDRS-line scoring.
(2) 15 letters or more improvement in ETDRS-letter scoring

(3) Three steps (0.3 logMAR) or more improvement in BA6 ${ }^{\circ}$

(4) Three steps (0.3 logMAR) or more improvement in GMA

\section{Statistics}

The anatomic and visual function measures described above were analyzed using SPSS Version 10.0 (SPSS Inc., Chicago, IL, USA). A Komogrov-Smirnov normality test was first performed and then Spearman's correlation coefficients were calculated to examine correlations between visual function measurements and the FP, FA, and OCT parameters. Logistic regression analysis was performed using the entry method. Each vision parameter, as the dependent variable was categorized into two categories, was representing successful vs unsuccessful outcomes. Exploratory variables included the change in the six FP/FA parameters and in the four OCT parameters.

\section{Results}

Among the 48 patients reviewed, the mean age was 75.4 years (range 50-98 years) with $41 \%$ female patients. Twenty-nine of the 50 eyes had undergone prior treatment with PDT, or with intravitreal injections of triamcinolone with/or pegaptanib. Before the bevacizumab treatment, the CNV was judged on FA to be classic in 12 eyes, predominantly classic in 11 eyes, minimally classic in 3 eyes, and occult in 24 eyes. The average number of bevicizumab injections was 4.5 with a range of 3-6. Mean duration of follow-up after initiation of treatment was 16 months (range 9-28 months).

\section{Association of functional vision with anatomic successful outcomes}

Table 1 presents the number of eyes that had successful visual outcomes according to each of the vision measurements following treatment. Functional success, defined by utilizing a $0.3 \log$ MAR equivalent improvement in the measurements, ranged from $12 \%$ of the eyes when measured with ETDRS-line chart acuity to $22 \%$ of the eye for GMA and $24 \%$ for $\mathrm{BA}^{\circ}$.

Table 1 Number of eyes showing a functional success (0.3 logMAR improvement) for each of the vision measurements evaluated

\begin{tabular}{ccccc}
\hline & ETDRS-line (\%) & ETDRS-letter (\%) & BA6 (\%) & GMA (\%) \\
\hline Number of eyes (n:50) & $7(14)$ & $6(12)$ & $12(24)$ & $11(22)$ \\
\hline
\end{tabular}

ETDRS, Early Treatment Diabetic Retinopathy Study; ETDRS-line, ETDRS-line scoring method; ETDRS-letter, ETDRS-total letter scoring method; BA6 ${ }^{\circ}$, best acuity within $6^{\circ}$ of fixation measured on acuity perimetry; GMA, global macular acuity. 


\section{Correlation of vision measurements with anatomic parameters}

Correlations using Spearman's correlation coefficients between each of the anatomic features and the visual function measurements are presented in Table 2. Before treatment, all vision measurements were observed to correlate only with the grading of RPE atrophy on FP $(P<0.05)$. The ETDRS line acuity was also correlated with grading of $\mathrm{CNV}$ fibrosis $(P<0.05)$. Following treatment, all vision measurements were again correlated with the RPE atrophy grade $(P<0.001)$. Both the ETDRS line acuity and the $\mathrm{BA} 6^{\circ}$ were also correlated with $\mathrm{CNV}$ thickness on OCT $(P<0.05)$. Both the BA6 ${ }^{\circ}$ and the GMA were also correlated with the grade of $\mathrm{CNV}$ fibrosis $(P<.001$ and $P<0.05$, respectively).

\section{Regression analysis comparing vision outcomes with change in anatomic parameters}

Logistic regression analysis was performed evaluating the outcome of each vision measurement as the dependent variable with the changes in the anatomic parameters as the independent variables (presented in

Table 2 Spearman's correlation coefficients between vision measurements and FA and OCT parameters prior to and following treatment

\begin{tabular}{lcc}
\hline $\begin{array}{l}\text { Vision measuring methods compared } \\
\text { with anatomic features }\end{array}$ & $\begin{array}{c}\text { Prior to } \\
R x(R)\end{array}$ & $\begin{array}{c}\text { Following } \\
R x(R)\end{array}$ \\
\hline ETDRS-line & & \\
Grade of CNVM fibrosis & $0.280^{\mathrm{a}}$ & 0.212 \\
Grade of RPE atrophy & $0.323^{\mathrm{a}}$ & $0.453^{\mathrm{b}}$ \\
CNVM thickness & 0.199 & $-0.383^{\mathrm{a}}$ \\
ETDRS-letter & & \\
Grade of CNVM fibrosis & -0.235 & 0.226 \\
Grade of RPE atrophy & $-0.318^{\mathrm{a}}$ & $-0.471^{\mathrm{b}}$ \\
CNVM thickness & -0.171 & $-0.385^{\mathrm{a}}$ \\
& & \\
BA6 & & \\
Grade of CNVM fibrosis & 0.255 & $0.447^{\mathrm{b}}$ \\
Grade of RPE atrophy & $0.292^{\mathrm{a}}$ & $0.515^{\mathrm{c}}$ \\
CNVM thickness & 0.196 & $0.313^{\mathrm{a}}$ \\
GMA & & \\
Grade of CNVM fibrosis & & \\
Grade of RPE atrophy & 0.255 & $0.338^{\mathrm{a}}$ \\
CNVM thickness & $0.292^{\mathrm{a}}$ & $0.298^{\mathrm{a}}$ \\
\hline
\end{tabular}

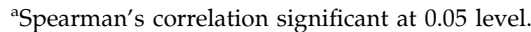

${ }^{\mathrm{b} C}$ Correlation is significant at 0.001 level.

${ }^{\mathrm{c}}$ Correlation is significant at 0.0001 level

$\mathrm{R}$, coefficient of correlation; $\mathrm{BA}^{\circ}$, central $6^{\circ}$ best visual acuity; GMA, global macular acuity; CNVM thickness, choroidal neovascular membrane thickness; ETDRS, Early Treatment Diabetic Retinopathy Study; $\mathrm{RPE}$, retina pigment epithelium; prior to Rx, prior to the first bevicizumab treatment; following $\mathrm{Rx}$, after the last bevicizumab treatment.
Table 3). The analysis showed no statistically significant relation between the ETDRS line acuity outcome and the change of any of the anatomic parameters. The ETDRS letter acuity outcome was associated only with the change in SRF thickness (adjusted $R^{2}=0.47$ ) as was the vision outcome of $\mathrm{BA}^{\circ}$ (adjusted $R^{2}=0.35$ ). The outcome of the GMA was associated with the combined change in SRF thickness, in CNV thickness, and in CNV fibrosis grade $\left(R^{2}=0.34\right)$

\section{Discussion}

This study has evaluated longitudinal associations between measurements of vision and changes in the anatomic parameters occurring on FP, FA, and OCT measurements in eyes undergoing bevicizumab monotherapy for AMD-CNV. Not surprisingly, pre-treatment vision parameters were associated primarily with the grade of RPE atrophy and post treatment with the grade of RPE atrophy, as well as with the grade of subretinal fibrosis.

Several earlier studies have reported that angiographic parameters, such as CNV diameter and area, or leakage area do not adequately explain the associated distance VA. However, Moutray ${ }^{7}$ in a cross-sectional study of 74 eyes, observed that the angiography parameters of $\mathrm{CNV}$ diameter, CNV area, and leakage area, showed significant correlations primarily with near acuity and contrast sensitivity, as well as weaker associations with distance VA. The correlations were strongest with the parametric descriptors of FA morphology with minimal association observed with OCT measurements. Other longitudinal studies, however, have indicated that OCT parameters, such as maximal retinal thickness, or foveal thickness were useful to explain VA outcomes with treatment. 5,6,16

The above apparent conflicting results suggest that vision in eyes with AMD CNV most likely is a complex result of many factors, including but not necessarily limited to, the duration of subretinal, sero-sanguinous fluid, the resultant degree of subretinal fibrosis, and the severity of RPE, as well as choriocapillaris atrophy, all of which are poorly evaluated on FP or FA and not at all on OCT. If SRF is resolved by treatment, the vision may not necessarily improve if there is progressive RPE and choriocapillaris atrophy, or significant fibrosis that on pathology specimens is associated with overlying full-thickness retinal atrophy. ${ }^{17,18}$

Visual acuity, even when measured with the improved $\log$ MAR-ETDRS chart, has been criticized as a poor evaluation of vision, especially in eyes with macular disease $^{19}$ for the reasons cited above. On central field perimetry using discriminated targets, acuities in normal eyes decline uniformly in all directions from fixation to approximately $20 / 100$ at $10^{\circ}$ eccentricity. (Central field 
Table 3 Regression model of the relations between change in each vision measurement and change in each morphologic parameter

\begin{tabular}{|c|c|c|c|c|c|}
\hline Vision measuring methods & Classification table (\%) & Nagelkerke $\left(\mathrm{R}^{2}\right)$ & $\beta$-Coefficient & $S E$ & P-value \\
\hline ETDRS-letter & 94 & 0.466 & & & \\
\hline SRF thickness & & & 0.018 & 0.009 & $0.038^{\mathrm{a}}$ \\
\hline$B A 6^{\circ}$ & 78 & 0.345 & & & \\
\hline SRF thickness & & & 0.013 & 0.007 & $0.05^{\mathrm{a}}$ \\
\hline$G M A$ & 76 & 0.336 & & & \\
\hline SRF thickness & & & 0.011 & 0.006 & $0.05^{\mathrm{a}}$ \\
\hline CNV thickness & & & 0.009 & 0.008 & $0.05^{\mathrm{a}}$ \\
\hline Grade of CNV fibrosis & & & -0.112 & 1.050 & $0.042^{\mathrm{a}}$ \\
\hline
\end{tabular}

${ }^{a}$ Correlation is significant at 0.05 level.

ETDRS-letter, ETDRS-total letter scoring method; $\mathrm{BA} 6^{\circ}$, central $6^{\circ}$ best visual acuity; CNV thickness, choroidal neovascular membrane thickness; grade of CNV fibrosis, grade of choroidal neovascular membrane fibrosis; GMA, global macular acuity; SRF thickness, Subretinal fluid thickness; SE, standard error.

perimetry of discriminated target I. Results for normal individuals using high contrast targets. Japanese Journal of Ophthalmology, submitted.). However, the field in eyes with macular disease shows irregular areas of variable but frequently severe depressions. ${ }^{19}$ When a person can scan each letter of the chart for several seconds and happens to center the letter on an area of better functionality (at the center of fixation or off-axis), the recorded vision is better than when another locus is used, but this leads to extremely variable results on repeated testing with a chart. ${ }^{19}$ Chart measurements of contrast sensitivity have been suggested as a measure that shows better association with visual function in QOL questionnaires for activities of daily living, but the same problems exist when using this chart test for an eye with macular disease. Central acuity field testing, in which the discriminated targets are presented for brief periods that simulate fixations normally required during routine activities, ${ }^{20-22}$ represents a better measurement of functional vision. The acuity perimeter provides discrimination information at multiple intercepts from fixation outward to $10^{\circ}$ eccentricity for targets of one contrast. Although the perimeter provides the capability for testing vision discrimination under a number of contrasts that simulate routine activities of daily living, in this study, we chose to test at a high contrast to compare it with ETDRS chart acuity. Among a number of available perimetry analyses, we chose two: (1) the bestthresholded acuity at any intercept within $6^{\circ}$ of fixation $\left(\mathrm{BA}^{\circ}\right)$, as extra-foveal-preferred retinal loci in eyes with macular disease occur primarily within this eccentricity ${ }^{23,24}$ and (2) an average of the acuities thresholded at all intercepts within a $10^{\circ}$ radius of fixation weighed inversely for eccentricity (GMA), a result that appears to represent an overall evaluation of macular function. In this study, the acuity field parameters seemed to provide an improved understanding of the outcomes of vision accompanying the pathologic changes occurring in the macula with AMD CNV.

In this study, only $12-14 \%$ of the eyes experienced an improvement of vision equivalent to $0.3 \log$ MAR on ETDRS chart testing (a three line improvement that was utilized to define functional success). This is considerably less than $38-76 \%$, reported earlier for bevacizumab monotherapy, of CNV in $\mathrm{AMD}^{2,3,9,10,25-33}$ eyes and may have been caused by the high number of eyes that had undergone and failed in prior treatment, which would potentially select for eyes with poorer outcomes. ${ }^{34}$ Nevertheless, when the same $0.3 \log$ MAR improvement criteria was used to define success in the acuity field indices, nearly twice the number of eyes experienced a successful vision outcome. Several reasons may explain this difference: (1) The improvement in function may have been greater at the more peripheral intercepts because the effect of the antiVEGF drug on the CNV is primarily at its peripheral margins, where the neovascularization is less mature and more porous, (2) the medication may reduce the scotoma size and sharpen the scotoma edge more effectively than improving its central density, and (3) the areas of more substantial focal improvement in function may be severely limited in their field area and, therefore, are not sampled by searching that occurs with chart testing (the eye cannot keep the target within the off-axis, narrow, clearer portion of the visual field).

In this study, two of the photography parameters, the degree of subretinal RPE and choriocapillaris atrophy along with subretinal fibrosis, were statistically correlated with all the clinical measurements of vision. The relationship was stronger with the acuity perimetry indices than with the ETDRS chart measurements. Correlations between the vision measurements and all the OCT parameters were less significant than those 
associated with the measurements acquired from FP and FA, suggesting that fundus pathology of RPE atrophy and CNV fibrosis are the most important parameters explaining vision, although this does not conclude that the OCT parameters should not be used in decisions of surveillance for re-treatment. Among the anatomic parameters evaluated in the logistic regression model, we observed that the change in SRF thickness was the most common parameter that explained the functional outcomes with treatment. In the logistic regression model, SRF thickness alone explained $47 \%$ of the variation in ETDRS letter acuity and $35 \%$ of the variation in $\mathrm{BA} 6^{\circ}$. The combination of change in SRF thickness, along with the change in CNV thickness and change in grade of CNV fibrosis together explained $34 \%$ of the variation in GMA. Among the evaluated vision parameters, those derived from acuity perimetry, the BA6 $6^{\circ}$ and GMA, seemed to be best associated with change in grade of CNV fibrosis, in SRF thickness, and in CNV thickness. This seems to be similar to the observations of Moutray, ${ }^{7}$ in which near acuity, a test of more global macular function, correlated best with anatomic measurements.

In this longitudinal study of eyes treated with bevacizumab for CNV associated with AMD, there did not appear to be a unique anatomic marker for predicting visual outcomes. Similar to the observations of Moutray, ${ }^{7}$ both the photography and OCT measurements seemed to compliment one another and both were required to understand the impact of retinal pathology on vision and for evaluating the treatment of AMD.

We recognize that more than one of the anatomic variables evaluated may have significant cooperative effects on visual outcomes at the same time, but the retrospective nature of this pilot study and the small numbers of eyes prevent a substantial multivariate analysis. Nevertheless, the outcomes reported here should be considered when designing studies of new therapies for AMD and for understanding the changes in macular pathology, as well as visual function that results. We also recognize that future studies of AMD treatment should use central field acuity perimetry as a measure of more global macular function and that such perimetry should also be conducted under reduced contrast conditions that mimic normal activities of daily living for the better understanding of the visual function under such conditions.

\section{Acknowledgements}

This study was supported by TUBITAK.

\section{References}

1 Klein R, Klein BE, Jensen SC, Meuer SM. The five-year incidence and progression of age-related maculopathy: the Beaver Dam Eye Study. Ophthalmology 1997; 104: $7-21$.

2 Avery RL, Pieramici DJ, Rabena MD, Castellarin AA, Nasir MA, Giust MJ. Intravitreal bevacizumab (Avastin) for neovascular age-related macular dejeneration. Ophthalmology 2006; 113: 363-372.

3 Bashshur ZF, Bazarbachi A, Schakal A, Haddad ZA, El Haibi CP, Noureddin BN. Intravitreal bevacizumab for the management of choroidal neovascularization in age-related macular dejeneration. Am J Ophthalmol 2006; 142: 1-9.

4 Augustin AJ, Puls S, Offermann I. Triple therapy for choroidal neovascularization due to age-related macular degeneration: verteporfin PDT, bevacizumab, and dexamethasone. Retina 2007; 27: 133-140.

5 Krebs I, Binder S, Stolba U, Schmid K, Glittenberg C, Brannath $\mathrm{W}$ et al. Optical coherence tomography guided retreatment of photodynamic therapy. Br J Ophthalmol 2005; 89: 1184-1187.

6 Sahni J, Stanga P, Wong D, Harding S. Optical coherence tomography in photodynamic therapy for subfoveal choroidal neovascularisation secondary to age related macular degeneration: a cross sectional study. Br J Ophthalmol 2005; 89: 316-320.

7 Moutary T, Alarbi M, Mahon G, Stevenson M, Chakravarthy U. Relationships between clinical measures of visual function, fluorescein angiographic and optical coherence tomography features in patients with subfoveal choroidal neovascularization. Br.J. Ophthalmol 2008; 92: 361-364.

8 Hee MR, Baumal CR, Puliafito CA, Duker JS, Reichel E, Wilkins JR et al. Optical coherence tomography of agerelated macular degeneration and choroidal neovascularization. Ophthalmology 1996; 10: 1260-1270.

9 Chen CY, Wong JY, Heriot WJ et al. Intravitreal bevacizumab (Avastin) for neovascular age-related macular degeneration: a short-term study. Am J Ophthalmol 2007; 143: 510-512.

10 Yoganathan P, Deramo VA, Lai JC, Tibrewala RK, Fastenberg DM. Visual improvement following intravitreal bevacizumab (Avastin) in exudative age-related macular degeneration. Retina 2006; 26: 994-998.

11 Salinas-Alaman A, Garcia-Layana A, Maldonado MJ, Sainz-Gómez C, Alvárez-Vidal A. Using optical coherence tomography to monitor photodynamic therapy in age-related macular degeneration. Am J Ophthalmol 2005; 140: $23-28$.

12 Ting TD, Oh M, Cox TA, Meyer CH, Toth CA. Decreased visual acuity associated with cystoid macular edema in neovascular age-related macular degeneration. Arch Ophthalmol 2002; 120: 731-737.

13 Sinclair SH, Alaniz R, Presti P. Laser treatment of diabetic macular edema: comparison of ETDRS-level treatment with threshold-level treatment by using high-contrast discriminant central visual field testing. Semin Ophthalmol 1999; 14(4): 214-222.

14 Ferris III FL, Kassoff A, Bresnick GH, Bailey I. New visual acuity charts for clinical research. Am J Ophthalmol 1982; 94: 91-96.

15 Hee MR, Izatt JA, Swanson EA, Huang D, Schuman JS, Lin CP et al. Optical coherence tomography of the human retina. Arch Ophthalmol 1995; 113: 325-332. 
16 Kliffen M, Sharma HS, Mooy CM, Kerkvliet S, de Jong PT. Increased expression of angiogenic growth factors in agerelated maculopathy. Br J Ophthalmol 1997; 81: 154-162.

17 Zarbin MA. Current concepts in the pathogenesis of agerelated macular degeneration. Arch Ophthalmol 2004; 122: 598-614.

18 Kliffen M, Van der Schaft T, Mooy CM, De Jong PT. Morphologic changes in age-related maculopathy. Microsc Res Tech 1997; 36: 106-122.

19 Gonzalez E, Tarita-Nistor L, Markowitz SN, Steinbach MJ. Computer-based test to measure optimal visual acuity in age-related macular degeneration. Invest Ophthalmol Visl Sci 2007; 48: 4838-4845.

20 Moore T. Shape representations and visual guidance of saccadic eye movements. Science 1999; 285: 5435.

21 Hoffman JE. The role of attention in saccadic eye movements. Percept Psychophys 1995; 5: 787-795.

22 Vogel GL. Saccadic eye movements: theory testing and therapy. J Behav Optom 1995; 6(1): 3-12.

23 Sunness J, Applegate C, Haselwood D, Rubin GS. Fixation patterns and rading rates in eyes with central scotomas from advanced atrophic age-related macular degeneration and Stargardt disease. Ophthalmology 1996; 103: 1458-1466.

24 Sunness J, Applegate C. Long-term follow-up of fixation patterns in eyes with central scotomas from geographic trophy that is associated with age-related macular degeration. Am J Ophthalmol 2005; 140: 1085-1093.

25 Spaide RF, Laud K, Fine HF, Klancnik Jr JM, Meyerle CB, Yannuzzi LA et al. Intravitreal bevacizumab treatment of choroidal neovascularization secondary to age-related macular degeneration. Retina 2006; 26: 383-390.

26 Aggio FB, Farah ME, Silva WC, Melo GB. Intravitreal bevacizumab for exudative age-related macular degeneration after multiple treatments. Graefes Arch Clin Exp Ophthalmol 2007; 245: 215-220.
27 Rich RM, Rosenfeld PJ, Puliafito CA, Dubovy SR, Davis JL, Flynn Jr HW et al. Short-term safety and efficacy of intravitreal bevacizumab (Avastin) for neovascular agerelated macular degeneration. Retina 2006; 26: 495-511.

28 Emerson MV, Lauer AK, Flaxel CJ, Wilson DJ, Francis PJ, Stout JT et al. Intravitreal bevacizumab (Avastin) treatment of neovascular age-related macular degeneration. Retina 2007; 27: 439-444.

29 Lazic R, Gabric N. Intravitreally administered bevacizumab (Avastin) in minimally classic and occult choroidal neovascularization secondary to age-related macular degeneration. Graefes Arch Clin Exp Ophthalmol 2007; 245: 68-73.

30 Pedersen R, Soliman W, Lund-Andersen H, Larsen M. Treatment of choroidal neovascularization using intravitreal bevacizumab. Acta Ophthalmol Scand 2007; 85: 526-533.

31 Jonas JB, Libondi T, Ihloff AK, Harder B, Kreissig I, Schlichtenbrede $\mathrm{F}$ et al. Visual acuity change after intravitreal bevacizumab for exudative agerelated macular degeneration in relation to subfoveal membrane type. Acta Ophthalmol Scand 2007; 85: 563-565.

32 Aisenbrey S, Ziemssen F, Völker M, Gelisken F, Szurman P, Jaissle $\mathrm{G}$ et al. Intravitreal bevacizumab (Avastin) for occult choroidal neovascularization in age-related macular degeneration. Graefes Arch Clin Exp Ophthalmol 2007; 247: 941-948.

33 Abraham-Marin ML, Cortes-Luna CF, Alvarez-Rivera G, Hernández-Rojas M, Quiroz-Mercado H, Morales-Cantón V. Intravitreal bevacizumab therapy for neovascular agerelated macular degeneration: a pilot study. Graefes Arch Clin Exp Ophthalmol 2007; 245: 651-655.

34 Levy J, Shneck M, Rosen S, Klemperer I, Rand D, Weinstein $\mathrm{O}$ et al. Intravitreal bevacizumab (avastin) for subfoveal neovascular age-related macular degeneration. Int Ophthalmol 2008. [Epub ahead of print]. 